Novi Chavarria, E. (2019). Il teatro delle e per le monache (Napoli, secolo XVIII). Italica Wratislaviensia, 10(2), 119-133.

DOI: http://dx.doi.org/10.15804/IW.2019.10.1.20

\author{
Elisa Novi Chavarria \\ Università degli Studi del Molise \\ novi@unimol.it \\ ORCID: 0000-0002-8531-0563
}

\title{
IL TEATRO DELLE E PER LE MONACHE (NAPOLI, SECOLO XVIII)
}

\section{THE THEATRE OF AND FOR THE NUNS (NAPLES, $18^{\mathrm{TH}}$ CENTURY)}

\begin{abstract}
Recent studies have focused on the musical environment and the theatre in female monasteries of many Italian cities between the $16^{\text {th }}$ and $18^{\text {th }}$ centuries. These art forms became famous as forms of entertainment in travel literature and in the chronicles of the time but were forbidden in the age of the Counter-Reformation. However, the theatrical performances, both in prose and in music, enjoyed enormous success and spread in male and female monasteries. As of the $17^{\text {th }}$ century, if not even earlier, travellers from half of Europe arrived in Naples, attracted by the excellence of the musical and theatrical performances that they could enjoy in the monasteries of the city.

This essay aims to reconstruct the times, the modalities, and the contents of the theatrical offerings in the female monasteries of Naples at the beginning of the $18^{\text {th }}$ century, all of which are still unknown today. In particular, the case of the Franciscan monastery of St Chiara will be examined. Through the patronage of Queen Maria Amalia, musical and theatrical performances played an active leading role in the configuration of a specific theatrical type and taste and increased the education of the nuns and young women who were educated in the monastery, representing and legitimising new feelings and sensibilities. The religious women found a way to talk of their feelings and concerns together; they forged relationships even with the environments outside of the monastery and especially with the Queen's court and with the courts of the aristocratic palaces of their families of origin.
\end{abstract}

Keywords: theatre, music, female monasteries, Naples, $18^{\text {th }}$ century 


\section{UNA “BURLETTA” PER MONACHE} ella stagione teatrale 1984/85 Roberto De Simone portò in scena
con la compagnia Ente Teatro Cronaca Le religiose alla moda, libero adattamento in musica di una commedia in tre atti composta nel 1768 dall'avvocato napoletano Gioacchino Dandolfo, già nota per l'attenzione che vi aveva riposto Benedetto Croce. Questi, nel 1898, ne aveva pubblicato, infatti, la trascrizione di alcuni brani dal manoscritto originale, conosciuto in diverse copie ${ }^{1}$. La sceneggiatura di De Simone si avvaleva, invece, della trascrizione integrale fornita da Franco Carmelo Greco (Scognamiglio, 2007, p. 111).

Lo spettacolo rimase in cartellone a Napoli per diverso tempo. Nell'ottobre del 2009, e di nuovo a giugno 2015, la commedia è stata riportata in scena, nel teatro van Westerhout di Mola di Bari, dal regista Paolo Panaro, anche stavolta con notevole successo di pubblico, come riportato dalla stampa non solo locale².

La trama della commedia - scriveva Croce - si basa sulle gelosie e insofferenze reciproche delle religiose di uno dei tanti monasteri napoletani dell'epoca che gareggiavano tra loro per conquistarsi la predilezione del padre confessore con piccoli doni e velate compiacenze, tra le vicendevoli insinuazioni di maldicenze e malattie immaginarie, scrupoli esasperati e l'accondiscendenza di quanti, dentro e fuori le mura del convento, entravano in relazione con loro. In alcune scene la commedia scandaglia anche l'universo emozionale delle converse, maltrattate dalle monache coriste, quasi sempre di estrazione aristocratica, che in virtù dei loro privilegi di nascita e lo status gerarchico di cui godevano nel monastero relegavano le loro più umili consorelle ai lavori domestici più pesanti, costringendole a pulizie meticolose, e "inutili" secondo l'autore, a fare e disfare letti secondo complicate geometrie o a recapita-

${ }^{1}$ Croce ripubblicò il suo scritto nel 1954 (vol. III, pp. 69-73). Esso ora si legge nella nuova edizione 1992, pp. 219 sgg.

2 https://ricerca.repubblica.it/repubblica/archivio/repubblica/2009/10/03/le-religiose-alla-moda-panaro-al-van.html; https://ricerca.repubblica.it/repubblica/archivio/ repubblica/2015/06/25/panaro-porta-in-scena-religiose-alla-modaBari10.html [data di consultazione: 24 ottobre 2018]. 
re dolci da loro confezionati e bigliettini in giro per la città anche sotto le più avverse condizioni meteorologiche. In breve, un ritratto ironico ed impietoso di quel mondo rappresentato dal Settecento monastico femminile biasimato contemporaneamente, per altri versi, dal pensiero di riformatori e anticurialisti napoletani, e non solo napoletani, che ne ravvisavano il coacervo di interessi privati e vessazioni familiari nei confronti delle donne, oltre che l'oneroso storno di importanti risorse economiche, rappresentato dal fenomeno delle doti monastiche e della manomorta ecclesiastica, verso obiettivi del tutto improduttivi ${ }^{3}$.

Sia nell'allestimento di De Simone, che in quello di Panaro, i ruoli delle religiose sono stati affidati ad attori uomini in abiti femminili, secondo una consuetudine invalsa al tempo in cui la commedia fu scritta, ma biasimata da molti tra gli stessi religiosi, per «essere - come scrisse, per esempio, il gesuita Cesare Calino nel 1717 - uno spettacolo assai più abominevole vedere un sacerdote coprire la cherica sacerdotale con un cimiero donnesco e farsi vedere in teatro a numero grande di spettatori con busto e sottano» (vol. I, p. 511) ${ }^{4}$.

Sul profilo di Gioacchino Dandolfo (1724-1792), l'autore della commedia in questione, originario di Conca dei Marini, una piccola località marittima vicino ad Amalfi, compositore di commedie sia interamente scritte, sia di soggetti a canovaccio, sulla sua ampia produzione di testi per il teatro per lo più fondata sulla pratica della "commistione tra dilettantismo e professionismo", ha scritto un saggio chiarificatore, a mio avviso, dal punto di vista filologico Ettore Stizzo a cui mi sento senz'altro di rinviare. Il magistero letterario e teatrale, per molti versi anche innovatore, del Dandolfo - egli ha notato - si concentra tutto nel settimo decennio del Settecento. Non è stato possibile ricostruire nel dettaglio il suo entourage o la rete dei suoi possibili committenti, né la cronologia delle composizioni, dei debutti teatrali o della pubblicazione delle sue opere. Grazie a notizie indirette, desunte per lo più da crona-

3 Un vero e proprio atto di accusa del giurisdizionalismo napoletano nei confronti dell'intero sistema di accesso ai monasteri fu raccolto da Vargas Machuca, 1745. I termini del dibattito sono stati analizzati da Campanelli, 2012, pp. 25-44.

4 Sul disciplinamento delle donne nel teatro si rinvia a Majorana, 1996. 
che e Avvisi, sappiamo però che altre sue opere furono rappresentate nel convento dei padri Celestini di S. Pietro a Maiella, godendo di ripetute presenze sulle scene e ampio successo (Stizzo, 2001).

La commedia Le religiose alla moda certamente fu scritta per essere rappresentata in un monastero femminile, come si evince dal sottotitolo del manoscritto originario, che recita appunto: Burletta per monache. Non sappiamo per quale monastero il Dandolfo l'avesse scritta, né dove fu rappresentata. Le diverse stesure con cui il testo è sopraggiunto fino a noi - Stizzo ne ha contate ben dieci - ci inducono in ogni caso a ritenere che essa dovette andare più volte in scena anche nel corso del secolo XVIII. Sappiamo per certo, d'altronde, che spettacoli teatrali, sia in prosa che soprattutto in musica, avevano una enorme fortuna e diffusione nei monasteri maschili e femminili napoletani, e non da allora ${ }^{5}$.

\section{MUSICA E TEATRO IN MONASTERO: UN'ANTICA CONSUETUDINE}

Sin dal secolo XVII, se non da addirittura prima, Napoli aveva richiamato viaggiatori provenienti da mezza Europa anche in virtù della eccellenza delle esecuzioni canore e musicali di cui era possibile fruire nelle chiese dei monasteri femminili della città. Canto e teatro delle religiose erano offerti e fruiti dalla città e dai viaggiatori stranieri in visita in ripetuti momenti del calendario liturgico. Era usanza in molti monasteri, in occasione delle festività dei santi o delle cerimonie per la vestizione delle novizie, mettere in scena, infatti, delle sacre rappresentazioni, concerti polifonici o melodrammi musicali in cui le monache potevano fare sfoggio di virtuosismi canori e delle loro abilità musicali (Fiore, 2015). Vi eccellevano, nel Seicento, particolarmente le monache di S. Maria Donnalbina, come quella suor Isabella de Ligoro, dalla cui cella, nel 1618, l'arcivescovo Carafa sequestrò spartiti e strumenti musicali. Di molti di questi testi e brani musicali le monache erano spesso autrici e committenti, come per La regina Ester, un'opera in musica di Giaco-

${ }^{5}$ Il teatro dei santi e della devozione, che aveva nei conventi il suo scenario privilegiato, contò a Napoli anche su un'ampia produzione letteraria. Cfr. Brindicci, 2007. 
mo Badiale allestita nel convento della SS. Trinità nel 1691. Spese per musici, cantori e maestri di canto, quasi sempre reclutati tra i canonici della cattedrale o i maestri della Cappella maggiore del Palazzo reale, sono documentate per il secolo XVII per le comunità religiose femminili di S. Chiara, S. Maria della Provvidenza, S. Gregorio Armeno, S. Geronimo, S. Francesco dell'Osservanza e della SS. Trinità (Novi Chavarria, 2009, pp. 148-149). Per gli anni 1726-1737 disponiamo poi dei regesti delle polizze bancarie reperite nei giornali di cassa dell'Archivio del Banco di Napoli, edite a cura di Francesco Cotticelli e Paologiovanni Maione, ove pure risultano diffusamente attestati i rilevanti importi destinati da numerosi monasteri napoletani alla retribuzione di musici, organisti, maestri di cappella e maestri di canto per le religiose (Cotticelli, Maione, 2015).

Come è noto la normativa tridentina aveva proibito tali pratiche. A Napoli, i sinodi del 1646 e del 1649 vietarono alle monache di rappresentare commedie, anche se di carattere sacro. Eppure - ed è solo un esempio - il 15 maggio 1650, il padre guardiano del monastero di S. Chiara permise alle monache di recitare, nella chiesa interna, una commedia con nove personaggi in abito secolare cui egli stesso assistette insieme a molti altri suoi confratelli (Russo, 1970, pp. 99-103). Nel 1726, i decreti del sinodo indetto dall'arcivescovo Francesco Pignatelli tornarono a vietare il canto alle monache, e in particolare il canto figurato, ma le monache mantennero ugualmente un'incisiva presenza sul palcoscenico della vita civica, cantavano e cantavano anche in canto figurato. L'abate senese Giovan Nicola Bandiera, venuto da Roma a Napoli proprio in quell'anno, nel 1726, raccontò come fosse uso in città recarsi «a sentir gli offici a' monasteri di monache, che per la verità generalmente - egli riferiva - cantano egregiamente; è però incredibile» - aggiungeva - «la pratica e l'invito che fanno i cicisbei delle monache canterine, e la cosa si riduce a tale che si sentono i viva come alle cantatrici de' teatri» (Strazzullo, 1978, pp. 14, 45). Nel monastero di S. Chiara, di giurisdizione regia e pertanto esente dal controllo dell'autorità episcopale, in quegli anni furono allestiti concerti con finanche 80 musicisti (Fiore, 2015b). Con 1'arrivo della regina Maria Amalia di Sassonia, sposa di Carlo di Borbone nel 1738, che le prese sotto la sua 
personale protezione, le monache di S. Chiara avviarono anche una pregevole ristrutturazione degli spazi comuni del monastero, in primis del giardino e del chiostro, affidando la direzione dei lavori all'architetto Domenico Antonio Vaccaro. Questi realizzò, tra le altre, il magnifico chiostro di 74 colonne di forma ottagonale ricoperte da maioliche dipinte con fogliami che è a molti noto, dove secondo la coeva moda francese dei giardini la passeggiata è intervallata da numerose sedute a forma di canapès, anch'esse maiolicate, che restituivano alle religiose la dimensione della sociabilità aristocratica vissuta nei palazzi delle loro famiglie d'origine (Novi Chavarria, 2009, pp. 96-98) ${ }^{6}$. Uno spazio mondano trovava così posto negli ambienti della clausura e nella solennità del chiostro, dilatandosi fin quasi a ricongiungersi con l'esterno. Non è escluso che questo spazio per così dire "intermedio" tra la clausura e il mondo esterno abbia potuto ospitare allestimenti musicali, ricreazioni e altre forme di spettacolo destinate anche a un pubblico estraneo al monastero. Le fonti del tempo parlano spesso di rappresentazioni e recite aperte al pubblico, cui poterono certamente assistere le famiglie delle monache o le religiose provenienti da altri monasteri. La tradizione teatrale conventuale si alimentava di norma, infatti, attraverso i contatti fra le diverse comunità femminili, non senza attingere motivi e forme dal teatro profano grazie ai continui scambi con l'ambiente sociale e culturale del tempo. Le monache mettevano in scena testi altrui o aggiornavano canovacci di storie tradizionali, oppure scrivevano commedie originali. Si ha notizia, per esempio, che a Roma, nel 1770, le religiose scalze di $\mathrm{S}$. Teresa assistettero alla recita preparata dalle educande del monastero delle Barberine della SS. Incarnazione ${ }^{7}$.

6 Cfr. Anche Manzo, 2014; Bisogno, Comes, 2018.

7 Nel Diario del monastero, pubblicato da Lirosi, Abbatelli, Palombo, 2016, p. 415, si legge: «A di 24 detto [1770]. Con licenza di nostro sig.re Clemente XIIII felicemente regnante e de nostri superiori le religiose scalze di s. Teresa entrarono in questo giorno nel nostro monastero per sentire la recita della commedia, che si faceva dalle nostre educande, per il che circa il mezzo giorno si aprì la porta consueta ed all'Ave Maria tornate le suddette religiose al loro monastero si serrò secondo il solito». 


\section{NAPOLI NEL SETTECENTO}

A Napoli, per antica consuetudine, durante il carnevale si rappresentavano drammi, oratori e commedie innanzi alle porte dei monasteri, per particolare divertimento di frati e monache. Recite aperte al pubblico venivano allestite nei conventi maschili di Monteoliveto, di S. Pietro Martire e presso la Congregazione dell'Oratorio di san Filippo Neri (Majorana, 2019). Nel monastero delle francescane di S. Chiara, dove tali pratiche pure erano consolidate da tempo, nel libro dei conti relativi al triennio 1739-1742 troviamo annotate spese straordinarie per "pietanze" offerte in occasione del «divertimento dato col permesso del sig. Cardinale protettore di ben sette operette tra spirituali e morali alla porta»-si legge nel documento - «(in quello spazio "intermedio" che crediamo per l'appunto di aver potuto individuare) per divertimento innocente in tutto il triennio: anzi la prima di esse ritrovata composta propriamente per noi in musica con prologo e stampa de' libretti, e l'altre tutte con intermezzi ed introduzione in musica» (Prota Giurleo, 1927, p. 66).

Sette spettacoli in tre anni, dal 1739 al 1742, quindi, realizzati presso il monastero di s. Chiara, seguiti da un banchetto più o meno suntuoso, andarono ad arricchire la fluidità dell'offerta teatrale della città di Napoli in quegli anni. Da questo punto di vista le pratiche artistiche e culturali dei monasteri femminili caratterizzarono e contribuirono a valorizzare il primato di Napoli nel teatro europeo. Esse parteciparono di quella vivacità e densità dell'offerta teatrale napoletana di luoghi, allestimenti e linguaggi per cui la città fu giustamente celebrata anche all'estero, di quel vero e proprio «dedalo teatrale partenopeo», come lo ha definito di recente Teresa Megale (2017), che finì con il legare indissolubilmente l'immagine e la percezione di Napoli a quella di «città-teatro»" ${ }^{8}$ Si trattò di un'offerta scenica in prosa e in musica allestita a corte, nei teatri cosiddetti minori, nei palazzi aristocratici, negli spazi provvisori e, nel caso dei monasteri femminili, forse meno eclatante ma ugualmente incisiva nelle consuetudini e nell'immaginario collettivo. Ne segnarono la

8 Sul variegato sistema teatrale napoletano nel Sei-Settecento si vedano gli studi di Cotticelli, Maione 1996, 2009. 
presenza sul territorio urbano della capitale e del Regno di Napoli, conferendo alla vita claustrale una proiezione pubblica e al chiostro ulteriori forme di prestigio e accrescimento delle proprie risorse simboliche e materiali. In certi casi - come si è detto - le rappresentazioni in musica e in prosa date nei monasteri femminili raggiunsero tra l'altro livelli di assoluta eccellenza, al punto da costituire motivo di vanto e di gloria per tutta la città.

Sono aspetti questi che gli studi di Gabriella Zarri (Zarri, Pomata, 2005) e, rispettivamente, di Robert Kendrick, Craig Monson e Colleen Reardon, per la musica, e di Elissa Weaver, per il teatro monastico femminili hanno largamente considerato e approfondito. Monson ha fatto luce sull'ambiente musicale monastico femminile di molte città italiane tra Cinque e Settecento, su alcune monache cantanti, strumentiste e compositrici di talento i cui idiomi musicali furono in certi casi anche straordinariamente moderni, sulla professionalità delle monache organiste, per esempio, e sul numero straordinariamente alto degli organi presenti nei monasteri femminili di Ferrara e Bologna (Monson, 2010). Si deve a Kendrick la riscoperta di altre religiose che furono anche loro compositrici talentuose e le cui musiche egli ha studiato all'interno della tradizione musicale della Milano al tempo dei due cardinali Borromeo e, in altri casi, documentando le finalità spirituali delle loro composizioni, spesso declinate in una teatralità totalmente vocale (Kendrick, 1996) ${ }^{9}$. Elissa Weaver ha avuto il merito di conferire spessore documentario alla fama di cui godevano - come abbiamo visto - nella letteratura da viaggio e nella memorialistica cittadine le commedie che venivano rappresentate nei monasteri femminili. In tutta Italia, e probabilmente in tutto il mondo cattolico, le monache durante il carnevale, per la vestizione delle novizie e in altre occasioni festive allestivano commedie, drammi e opere in musica sulla base di testi già noti o che venivano composti dalle stesse religiose al duplice scopo dell'intrattenimento spirituale e dell'apprendimento, e cioè per divertire e istruire le consorelle. A differenza di altre tipologie di scrittura monastica, come la memorialistica

9 Per la musica nei monasteri senesi si veda Reardon, 2002 e, per Venezia, Mantioni, 2017. 
o la biografia spirituale che lasciavano poco spazio alle citazioni culturali, tali testi rivelano spesso una conoscenza nient'affatto superficiale della letteratura classica e umanistico-rinascimentale (Weaver 2002; 2009).

Per tornare al caso del teatro monastico napoletano, nell'alveo di una ricerca che - è bene sottolineare - resta ancora in gran parte da fare, tra i materiali scenici che dovettero essere stati allestiti nel monastero di S. Chiara prima e, in particolare, durante l'abadessato di Ippolita Carmignano (1739-1742) grazie al matronage della regina Maria Amalia, segnalo: Il cielo in terra per la nascita del Redentore, un testo pubblicato postumo in occasione della sua rappresentazione in S. Chiara nel 1738, opera di Ettore Calcolona, lo pseudonimo con cui il canonico Carlo Celano, vissuto tra il 1617 e il 1693, più noto come autore di una delle più famose guide artistiche della città di Napoli, la celeberrima $L e$ Notizie del bello, dell'antico e del curioso della città di Napoli, firmò le oltre venti commedie del suo ricco repertorio di autore teatrale. Portata in scena più volte e più volte data pure alle stampe ${ }^{10}, 1^{\prime}$ operetta veniva ora allestita per l'onesto divertimento delle religiose di S. Chiara in segno anche di celebrazione dell'operato della badessa uscente, D. Aurelia Branci. Etichettato per lo più come un adattatore di temi cervantini e traduttore dal teatro spagnolo in quello italiano (Vaiopoulos, 2003), Celano/Calcolona fu in realtà ai suoi tempi, come la critica più recente ha evidenziato, un autore di successo e tra i maggiori esponenti del teatro barocco napoletano, solito scrivere anche per il pubblico del collegio dei gesuiti, di conventi e spazi aristocratici privati. Le sue commedie furono recitate con predilezione dalle compagnie comiche e ristampate frequentemente sia a Napoli che a Roma e Venezia. Sulla sua attività di compositore teatrale pesò, però, poi il giudizio inclemente di Benedetto Croce, che lo liquidò come «scimmiottatore alla spagnuola» e imitatore di testi altrui. Gli ammiratori coevi, e tra questi evidentemente anche le "nostre" monache di S. Chiara che commissionarono la messa in scena dell'operetta nel loro monastero, ne apprezzarono piuttosto il moralismo retorico, la capacità di fare coesistere i temi più diversi e, come nel

${ }^{10}$ Se ne conosce almeno un altro esemplare: Calcolona, 1698. 
caso del testo in questione, i riferimenti alla tradizione colta della poesia arcadica e bucolica ${ }^{11}$.

Durante il periodo di carnevale del 1738 fu rappresentato in S. Chiara anche il Dramma sacro di Giuseppe il Giusto, come ricreazione delle monache e delle educande che vivevano nel convento ${ }^{12}$. Sempre durante il carnevale, nel 1741, durante quindi l'abadessato della Carmignano, fu rappresentata l'opera buffa La tavernola abentorosa, libretto di Pietro Trinchera e musica di Carlo Cecere, violinista nel convento del Carmine maggiore di Napoli (Trinchera, 1740). L'opera, per la quale il suo autore incorse nelle maglie della censura ecclesiastica in seguito alla denuncia sportagli dall'arcivescovo Spinelli presso la segreteria dell'Ecclesiastico, era in effetti una proposta alquanto audace per un allestimento all'interno di un monastero. Vi si inscenavano, infatti, i loschi maneggi di un finto frate, tale Uzzacchio, un furbo che si faceva credere Eremita col nome di Fra' Macario per carpire elemosine e credito raggirando i più creduloni, una satira feroce, quindi, contro l'ipocrisia ammantata di devozione e la corruzione che resisteva ai margini della Chiesa, in generale, e di molte istituzioni ecclesiastiche, in particolare.

Non mi addentro nell'analisi di quest'opera e delle tormentate vicende biografiche del suo autore, sperimentatore di forme espressive anche al di fuori di schemi e convenzioni letterarie e che finì i suoi giorni suicida a causa dei debiti contratti nella sua attività di impresario teatrale. Anche in questo caso disponiamo di un' ampia letteratura sul tema cui è il caso senz'altro di rinviare ${ }^{13}$. Mi preme piuttosto sottolineare, in chiusura di queste mie note, la pluralità delle proposte teatrali messe in scena in quegli anni nei monasteri napoletani, la pluralità anche delle scelte organizzative e linguistiche, dialettali e non, che da un lato garantirono alle produzioni e alle imprese teatrali una ulteriore diversificazione dei circuiti di distribuzione e, dall'altro, offrirono al divertimento e alla istruzione delle religiose, e con loro del pubblico dei loro fami-

${ }^{11}$ Sul Celano come autore di teatro si vedano anche Russo, 1997, vol. I, p. 187; Marcello, 2019.

12 Giuseppe il Giusto (1738). Ho tratto la notizia dal Catalogo di commedie,1865, p. 14.

13 Si rimanda soprattutto agli studi di Cicali, 2000a, 2000b e 2015. 
liari che vi partecipavano, opportunità di confronto anche con esperienze marcatamente intellettuali e di dialogo con le punte più aggiornate del coevo teatro italiano. Vorrei sottolineare, inoltre, il ruolo che in tal senso esercitarono le monache, che come committenti di sceneggiature o come impresarie teatrali ebbero spesso un ruolo attivo e trainante nelle pratiche culturali cittadine e nella configurazione di uno specifico genere e gusto teatrali.

Spazio espressivo ed occasione per incrementare l'istruzione delle religiose e delle giovani che venivano educate nel monastero e offrire loro forme di "onesto divertimento", il teatro delle monache e per le monache fu anche e soprattutto il luogo in cui esse poterono rappresentare e legittimare sentimenti e nuove sensibilità. Fu uno spazio di configurazione della sociabilità aristocratica femminile, di reti di rapporti intessuti dentro e fuori il monastero, tra i monasteri e le corti, quella della Regina e le corti dei palazzi aristocratici delle famiglie di origine delle religiose, uno spazio in cui, al pari delle coeve accademie letterarie riservate ai loro nobili padri e fratelli, le religiose poterono coltivare rapporti e relazioni sociali, impreziosendole del valore aggiunto dato dalla elaborazione di più forme espressive e dalla condivisione della passione per il teatro e per la musica ${ }^{14}$. Basterà dire che il monastero di S. Chiara fu, nel Settecento, accanto ai cinque teatri cittadini, una delle sedi in cui più frequenti furono gli allestimenti scenici (Duraccio, 1998).

Ma non solo. Attraverso l'energia prorompente della ironia portata in scena nelle operette come quelle del Dandolfo o del Calcolona, le religiose poterono anche raccontarsi, fare affiorare e comunicare i propri sentimenti e inquietudini, e prendere così le opportune distanze emotive e ideologiche da quel sistema che se da un lato le vedeva recluse in convento, dall'altro apriva loro anche straordinarie opportunità di scelte e pratiche culturali. La relazione consapevole che molte di loro ebbero con il mondo del teatro e la sua capacità di trasformare e performare la realtà, e che la documentazione da me analizzata ha senz'altro messo

14 Analoghe considerazioni riguardo l'ambiente romano ha svolto Borello, 2003, pp. 102 sgg. 
in ulteriore risalto, avrebbe contribuito di lì a poco a scardinare dal di dentro quel medesimo sistema ${ }^{15}$.

\section{FONTI}

Calino, C. (1717). Discorsi scritturali, e morali ad utile trattenimento delle monache. Bologna: Ferdinando Pisarri.

Calcolona, C. (1698). Il cielo in terra per la nascita del Redentore. Rappresentazione sacra del Signor D. Ettorre Calcolona. Consagrata all'Illustriss. ed Eccellentiss. Sig. la Signora D. Aurora Sanseverino Caetano D'Aragona. Napoli: nella Stamperia di Carlo Troyse.

Calcolona, C. (1738). Il cielo in terra per la nascita del Redentore. Rappresentazione sacra del signor D. Ettorre Calcolona. Da rappresentarsi per innocente divertimento dalle dame religiose del regal monistero di S. Chiara in questo anno 1738. Napoli: nella stampa di Gennaro e Vincenzo Muzio.

Catalogo di commedie, drammi, tragedie, rappresentazioni sacre, favole pastorali, cantate etc. e di libri riguardanti il teatro. (1865). Napoli: A. Detken.

Giuseppe il Giusto (1738). Dramma sacro rappresentato nel carnevale del 1738 nel regal monastero di S. Chiara di Napoli, per divertimento di quelle dame religiose. Napoli: A. Parrino.

Trinchera, P. (1740?). La Tavernola Abentorosa. Melodramma addedecata lo muto llustre segnore D. Ghiennaro Finelli avocato napoletano. Napoli: s. t.

\section{BIBLIOGRAFIA}

Bisogno, S., \& Comes, F. (2018). Identità di genere nell'architettura religiosa del XVIII secolo: strutture monastiche e conventuali a Napoli. In C. Passetti \& L. Tufano (Eds.), Femminile e maschile nel Settecento (pp. 181-189). Firenze: Firenze University Press.

Borello, B. (2003). Trame sovrapposte. La socialità aristocratica e le reti di relazioni femminili a Roma (XVII-XVIII secolo). Napoli: Esi.

15 Sulla "svolta" emozionale del teatro e delle emozioni nel Settecento si rinvia a Plebani, 2012. 
Brindicci, M. (2007). Libri in scena. Editoria e teatro a Napoli nel secolo XVII. Napoli: Libreria Dante \& Descartes.

Campanelli, M. (2012). Monasteri di provincia (Capua secoli XVI-XIX). Milano: FrancoAngeli.

Cicali, G. (2000a). Fonti classiche e strategie retoriche di Pietro Trinchera. Il Castello di Elsinore, 13/39, 5-24.

Cicali, G. (2000b). Drammaturgia e fonti teatrali della Moneca fauza di Pietro Trinchera. Arte musica spettacolo. Annali del Dipartimento di Storia delle arti e dello spettacolo dell'Università di Firenze, 1/38, 113-133.

Cicali, G. (2015). Eduardo adattatore e "traduttore" di Trinchera. In N. De Blasi \& P. Sabbatino (Eds.), Eduardo De Filippo e il teatro del mondo (pp. 195-206). Milano: FrancoAngeli.

Cotticelli, F., \& Maione, P. (Eds.). (1996). Onesto divertimento ed allegria dei popoli. Materiali per una storia dello spettacolo a Napoli nel primo Settecento. Milano: Ricordi.

Cotticelli, F., \& Maione, P. (Eds.). (2009). Storia della musica e dello spettacolo a Napoli: il Settecento, 2 voll. Napoli: Edizioni Turchini.

Cotticelli, F., \& Maione, P. (2015). Spoglio delle polizze bancarie di interesse teatrale e musicale reperite nei giornali di cassa dell'Archivio del Banco di Napoli per gli anni 1726-1737. Studi pergolesiani. Pergolesi Studies, 9, cd-rom.

Croce, B. (1898). Vita dei monasteri napoletani. Da una commedia inedita del Settecento. Napoli nobilissima, 7, 163-167.

Croce, B. (1954). Aneddoti di varia letteratura. Bari: Laterza.

Croce, B. (1992). I teatri di Napoli. Dal Rinascimento alla fine del secolo decimottavo, a cura di G. Galasso. Milano: Adelphi.

Duraccio, M. (1998). Note sull'editoria teatrale napoletana nel Settecento. In A.M. Rao (Ed.), Editoria e cultura a Napoli nel XVIII secolo (pp. 683-696). Napoli: Liguori.

Fiore, A. (2015). Le carte degli antichi banchi e il panorama musicale e teatrale della Napoli di primo Settecento (1726-1736): musica nei chiostri femminili. Studi pergolesiani, 9, 679-714.

Fiore, A. (2015b). La tradizione musicale del monastero delle clarisse di Santa Chiara in Napoli. Rivista Italiana di Musicologia, 50, 33-60.

Lirosi, A., Abbatelli V., \& Palombo I. (Eds.). (2016). Un monastero di famiglia. Il diario delle barberine della SS. Incarnazione (secc. XVII-XVIII). Roma: Viella. 
Majorana, B. (1996). Finzioni, imitazioni, azioni: donne e teatro. In G. Zarri (Ed.), Donna disciplina creanza cristiana dal XV al XVII secolo. Studi e testi a stampa (pp. 121-139). Roma: Edizioni di storia e letteratura.

Majorana, B. (2019). Santi, bambini, attori. Francesco Gizzio e il teatro degli oratoriani a Napoli nel '600. Bruniana \& campanelliana, XXV/1, 201-216.

Mantioni, S. (2017). Monacazioni forzate e spazi di auto-affermazione femminile. Norma e prassi nel Serenissimo Dominio di età moderna. Roma: Gangemi.

Manzo, E. (2014). Tra sacro e profano: metamorfosi barocche nelle architetture di Domenico Antonio Vaccaro. Acta/Artis. Estudis d'Art Modern, 2, $13-23$.

Marcello, E.E. (2019). De la lectura al tablado. Antonio Matina, Carlo Celano, y otros estimadores de Lope en la Nápoles virreinal. Anuario Lope De Vega, 25, 122-142.

Megale, T. (2017). Tra mare e terra. Commedia dell'Arte nella Napoli spagnola (1575-1656). Roma: Bulzoni.

Monson, C.A. (2010). Nuns Behaving Badly. Tales of Music, Magic, Art, and Arson in the Convents of Italy. Chicago: University of Chicago Press.

Novi Chavarria, E. (2009). Sacro, pubblico e privato. Donne nei secoli XV-XVIII. Napoli: Guida.

Plebani, T. (2012). Un secolo di sentimenti: amori e conflitti generazionali nella Venezia del Settecento. Venezia: Istituto Veneto di Scienze, Lettere ed Arti.

Prota Giurleo, U. (1927). Nicola Logroscino "il dio dell'opera buffa": la vita e le opere. Napoli: Tip. Sangiovanni.

Kendrick, R.L. (1996). Celestial Sirens: Nuns and Their Music in Early Modern Milan. Oxford: Calendon Press.

Reardon, C. (2002). Holy Concord Within Sacred Walls: Nuns and Music in Siena, 1575-1700. Oxford: Oxford University Press.

Russo, C. (1970). I monasteri femminili di clausura a Napoli nel secolo XVII. Napoli: Università di Napoli, Istituto di Storia Medioevale e Moderna.

Russo, F. (1997). Riscoprire Carlo Celano. Proposta per una bibliografia. In V. De Gregorio (Ed.), Bibliologia e critica dantesca: saggi dedicati a Enzo Esposito (vol.1, pp. 187-206). Ravenna: Longo.

Scognamiglio, G. (2007). La scrittura che accende la scena: studi e testi teatrali da Bracco a Troisi. Napoli: Esi. 
Stizzo, E. (2001). Un commediografo napoletano del '700: Gioacchino Dandolfo. Critica letteraria, 112/3, 535-550.

Strazzullo, F. (1978). Dietro le grate del Divino Amore. Il Settecento religioso a Napoli. Napoli: Edizioni di Napoli Nobilissima.

Vaiopoulos, K. (2003). Temi cervantini a Napoli. Carlo Celano e la zingaretta. Firenze: Alinea.

Vargas Machuca, F. (1745). Dissertazione intorno la riforma degli abusi introdotti ne' Munisteri delle Monache per le doti e per le spese che vogliono dalle donzelle che vestono l'abito. Napoli.

Weaver, E.B. (2002). Convent Theatre in Early Modern Italy. Spiritual Fun and Learning for Women. Cambridge: Cambridge University Press.

Weaver, E.B. (2009). Scenes from Italian Convent Life: an Anthology of Convent Theatrical Texts and Contexts. Ravenna: Longo.

Zarri, G., \& Pomata, G. (Eds.). (2005). I monasteri femminili come centri di cultura fra Rinascimento e Barocco. Roma: Edizioni di storia e letteratura.

Riassunto: Studi recenti hanno fatto luce sull'ambiente musicale e sul teatro monastico femminile di molte città italiane tra Cinque e Settecento, conferendo spessore documentario alla fama di cui tali forme di spettacolo godevano nella letteratura da viaggio e nella memorialistica cittadine dell'epoca. Si trattava di pratiche culturali vietate dalla normativa post-tridentina, eppure spettacoli teatrali, sia in prosa che in musica, ebbero una enorme fortuna e diffusione nei monasteri maschili e femminili. Napoli, in particolare, sin dal secolo XVII, se non da addirittura prima, richiamò viaggiatori provenienti da mezza Europa anche in virtù della eccellenza delle esecuzioni musicali e teatrali di cui era possibile fruire nei monasteri della città. Il saggio prova a far luce su tempi, modalità e contenuti dell'offerta teatrale dei monasteri femminili napoletani agli inizi del secolo XVIII, in particolare del monastero delle francescane di S. Chiara che, grazie al patronage della regina Maria Amalia, pare abbia avuto un ruolo attivo e trainante nella configurazione di uno specifico genere e gusto teatrali volto a incrementare l'istruzione delle religiose e delle giovani che venivano educate nel monastero, ma anche a rappresentare e legittimare sentimenti e nuove sensibilità. Le religiose vi trovarono un modo per raccontarsi, fare affiorare e comunicare i propri sentimenti e inquietudini, attivare forme di sociabilità e di relazioni sociali dentro e fuori il monastero, tra i monasteri e le corti, quella della Regina e le corti dei palazzi aristocratici delle loro famiglie di origine.

Parole chiave: teatro, musica, monasteri femminili, Napoli, secolo XVIII 